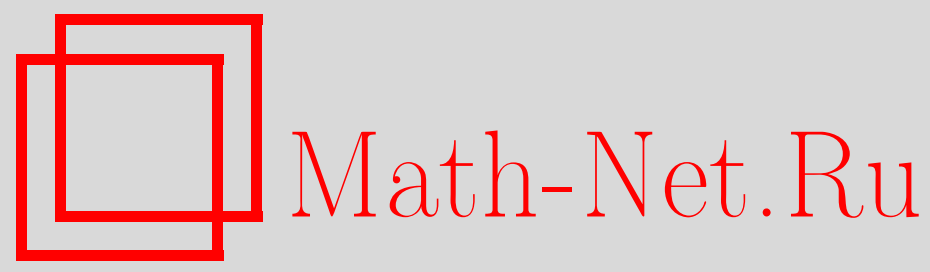

В. Л. Крепкогорский, Реализация интерполяционного метода Спарра в классах пространств гладких функций, $M a-$ тем. заметки, 2001, том 70, выпуск 4, 581-590

DOI: https://doi.org/10.4213/mzm770

Использование Общероссийского математического портала Math-Net.Ru подразумевает, что вы прочитали и согласны с пользовательским соглашением http://www.mathnet.ru/rus/agreement

Параметры загрузки:

IP: 54.172 .240 .79

26 апреля 2023 г., $17: 00: 54$

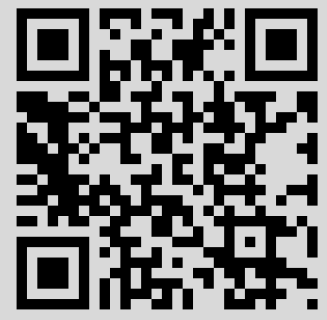




\section{РЕАЛИЗАЦИЯ ИНТЕРПОЛЯЦИОННОГО МЕТОДА СПАРРА В КЛАССАХ ПРОСТРАНСТВ ГЛАДКИХ ФУНКЦИЙ}

\section{В. Л. Крепкогорский}

Интерполяционный многомерный метод Спарра реализуется в пространствах Бесова $B_{p, q}^{s}$ и Лизоркина-Трибеля $F_{p, q}^{s}$. Показано, что в результате получаются пространства Бесова типа $B_{p, q,(q)}^{s}$. Сформулирована интерполяционная теорема для пространств Бесова, использующая слабые условия вида $T: F_{p, 1,(1)}^{s} \rightarrow F_{\widetilde{p}, \infty,(\infty)}^{\widetilde{s}}$.

Библиография: 8 названий.

В начале 70-х годов Спарром [1] был предложен многомерньй интерполяционньй метод, аналогичньй хорошо известному методу Петре. В отличие от функторов метода Петре функторы Спарра действуют не на пары, а на наборы из $n$ пространств. Различные обобщения результатов Спарра рассматривались в статьях [2]-[4]. Общая теория многомерных методов разработана достаточно глубоко, однако их применение вызьвает значительные трудности. Допустим, что мы применяем многомерный функтор к набору пространств из некоторого семейства. Какое пространство мы получим в результате? Ответ на этот вопрос известен только для немногих классов пространств. Например, Спарр реализовал свой метод в классе пространств типа Бесова $S_{p}^{\bar{s} q} B$ с векторным параметром $\bar{s}$ в случае, когда $p_{0}=p_{1}=p_{2}=\cdots=p_{n}$. Возможно, один из путей, который позволяет обойти возникающие при реализации трудности, - это использование теорем о стабильности (аналогов теоремы о реитерации). В статье Спарра приведены три теоремы о стабильности, в том числе очень полезные теоремы, связьвающие функторы различной размерности. Однако во всех этих теоремах содержится оговорка, что они справедливы только при условии вьполнения в данном классе пространств равенства $A_{\bar{\theta}, q ; K}=A_{\bar{\theta}, q ; J}$. Проверка этого условия представляет из себя нетривиальную задачу. Значительное продвижение в этом направлении достигнуто в статье Асекритовой и Кругляка [4], которые показали, что равенство $A_{\bar{\theta}, q ; K}=A_{\bar{\theta}, q ; J}$ вьполняется в семействах банаховых функциональных структур и их ретрактов.

1. Метод Спарра. Рассмотрим набор из $n+1$ банаховых пространств $\bar{A}=\left\{A_{0}, A_{1}\right.$, $\left.\ldots, A_{n}\right\}$. Для $a \in \Sigma(\bar{A})$ запишем

$$
K(\bar{t}, a, \bar{A})=\inf \left\{t_{0}\left\|a_{0}\left|A_{0}\left\|+t_{1}\right\| a_{1}\right| A_{1}\right\|+\cdots+t_{n}\left\|a_{n} \mid A_{n}\right\|\right\}
$$

где $a=a_{0}+a_{1}+\cdots+a_{n}$. 
Пусть $H_{+}^{n}$-множество всех векторов $\left(\lambda_{0}, \lambda_{1}, \ldots, \lambda_{n}\right)$, для которых $\sum \lambda_{j}=1, \lambda_{j}>0$. Допустим, что $\bar{\theta} \in H_{+}^{n}$ и $1 \leqslant q \leqslant \infty$. Тогда определим норму $\Phi_{\bar{\theta}, q}$ с помошью равенства

$$
\Phi_{\bar{\theta}, q}:=\left(\int_{\mathbb{R}_{+}^{n}}\left|t_{1}^{-\theta_{1}} \cdots t_{n}^{-\theta_{n}} \cdot f\left(1, t_{1}, t_{2}, \ldots, t_{n}\right)\right|^{q} \frac{d t_{1}}{t_{1}} \cdots \frac{d t_{n}}{t_{n}}\right)^{1 / q} .
$$

Рассмотрим три теоремы о стабильности метода Спарра [1]. Ecлu

Teоpema A. Пусть $\bar{\theta}^{i} \in H_{+}^{n}(i=0,1, \ldots, m), \bar{\theta} \in H_{+}^{n}, \bar{\lambda} \in H_{+}^{m} c \bar{\theta}=\sum_{i=0}^{m} \lambda_{i} \bar{\theta}^{i}$.

a) $\mathbb{R}^{n+1}$ совпадает с линейной оболочкой множества векторов $\left\{\bar{\theta}^{i}\right\}_{0}^{m}$;

b) $A_{\bar{\theta}, q ; K}=A_{\bar{\theta}, q ; J}$,

$m o\left(A_{\bar{\theta}^{0}, q_{0}}, A_{\bar{\theta}^{1}, q_{1}}, \ldots, A_{\bar{\theta}^{m}, q_{m}}\right)_{\bar{\lambda}, q}=A_{\bar{\theta}, q}$. Ecлu

Teоpema В. Пусть $\bar{\theta}^{i} \in H_{+}^{n}(i=0,1, \ldots, m), \bar{\theta} \in H_{+}^{n}, \bar{\lambda} \in H_{+}^{m} c \bar{\theta}=\sum_{i=0}^{m} \lambda_{i} \bar{\theta}^{i}$.

a) $1 / q=\sum_{i=0}^{m} \lambda_{i} / q_{i}$

b) $A_{\bar{\theta}, q ; K}=A_{\bar{\theta}, q ; J}$,

$m o\left(A_{\bar{\theta}^{0}, q_{0}}, A_{\bar{\theta}^{1}, q_{1}}, \ldots, A_{\bar{\theta}^{m}, q_{m}}\right)_{\bar{\lambda}, q}=A_{\bar{\theta}, q}$.

Теорема С. При $\bar{\theta} \in H_{+}^{n}, \bar{\theta}=\left(\theta_{0}, \theta_{1}, \ldots, \theta_{n}\right)$, имеет место равенство

$$
\left(\left(A_{0}, A_{n}\right)_{\bar{\eta}, q},\left(A_{1}, A_{n}\right)_{\bar{\eta}, q}, \ldots,\left(A_{n-1}, A_{n}\right)_{\bar{\eta}, q}\right)_{\bar{\lambda}, q}=A_{\bar{\theta}, q},
$$

əде

$$
\bar{\eta}=\left(\theta_{0}+\theta_{1}+\cdots+\theta_{n-1}, \theta_{n}\right), \quad \bar{\lambda}=\frac{\left(\theta_{0}, \theta_{1}, \ldots, \theta_{n-1}\right)}{\theta_{0}+\theta_{1}+\cdots+\theta_{n-1}} .
$$

В случае $n=1$ функторы метода Спарра совпадают с обычньми функторами метода Петре. Точнее, справедливо равенство

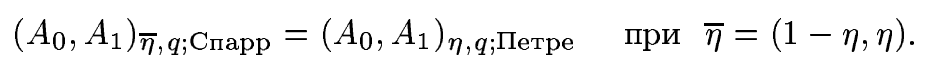

В дальнейшем мы будем отличать функторы Петре по скалярному первому параметру интерполяции (в данном случае это $\eta$ ).

Кроме того, будем использовать следующие интерполяционные формулы. Пусть $0<\theta<1,1 \leqslant q_{0}, q_{1}, q \leqslant \infty, 1<p_{0}, p_{1}, p<\infty,-\infty<s_{0}, s_{1}, s<\infty$,

$$
\left(F_{p, q_{0}}^{s_{0}}\left(\mathbb{R}^{n}\right), F_{p, q_{1}}^{s_{1}}\left(\mathbb{R}^{n}\right)\right)_{\theta, q}=B_{p, q}^{s}\left(\mathbb{R}^{n}\right)
$$

при $s_{0} \neq s_{1}$ и $s=(1-\theta) s_{0}+\theta s_{1}[5$, c. 223]

$$
\left(B_{p_{0}, q_{0}}^{s_{0}}\left(\mathbb{R}^{n}\right), B_{p_{1}, q_{1}}^{s_{1}}\left(\mathbb{R}^{n}\right)\right)_{\theta, q}=B_{p, q,(q)}^{s}\left(\mathbb{R}^{n}\right)
$$

при $p_{0} \neq p_{1}, s=(1-\theta) s_{0}+\theta s_{1}, 1 / p=(1-\theta) / p_{0}+\theta / p_{1}, 1 / q=(1-\theta) / q_{0}+\theta / q_{1}[5$, c. 218].

Если дополнительно $q=p$, то [5, c. 218]

$$
\left(B_{p_{0}, q_{0}}^{s_{0}}\left(\mathbb{R}^{n}\right), B_{p_{1}, q_{1}}^{s_{1}}\left(\mathbb{R}^{n}\right)\right)_{\theta, q}=B_{p, p}^{s}\left(\mathbb{R}^{n}\right)=B_{p}^{s}\left(\mathbb{R}^{n}\right) .
$$


2. Пространства Лизоркина-Трибеля. В этом пункте мы реализуем метод Спаррав классе пространств Лизоркина-Трибеля. Пространства Лизоркина-Трибеля $F_{p, q}^{s}$ и Бесова $B_{p, q}^{s}$ мы будем изображать точками координатной плоскости, где на одной из осей откладьвается величина $1 / p$, а на другой - величина $s$. Если данному пространству соответствует точка $M$ на плоскости, то будем обозначать это пространство $F_{(M), q}$ или $B_{(M), q}$.

Tеорема 1. Пусть $-\infty<s_{j}, s<\infty, 1<p<\infty, 1 \leqslant q_{j}, q \leqslant \infty, \bar{\theta} \in H_{+}^{2}, j=0,1,2$; при этом если два значения $s_{j}$ равны между собой, то пусть соответствующие $q_{j}$ также равны между собой. Положим

$$
s=\sum_{j=0}^{2} \theta_{j} s_{j}, \quad \frac{1}{p}=\sum_{j=0}^{2} \frac{\theta_{j}}{p_{j}}
$$

$Q(1 / p, s)$ - внутренняя точка треугольника $\Delta M_{j}\left(1 / p_{j}, s_{j}\right)$. Тогда

$$
\left(F_{p_{j}, q_{j}}^{s_{j}}\left(\mathbb{R}^{n}\right)\right)_{\bar{\theta}, q}=B_{p, q,(q)}^{s}\left(\mathbb{R}^{n}\right)
$$

Докажем сначала лемму.

ЛЕмма 1. Даны треугольники $\Delta M_{j}\left(1 / p_{j}, s_{j}\right)$ и $\Delta N_{j}\left(1 / \widetilde{p}_{j}, \widetilde{s}_{j}\right)$, гомотетичные относительно общей вершины $M_{2}=N_{2}\left(q_{2}=\widetilde{q}_{2}\right), \Delta N_{j} \subset \Delta M_{j}, Q(1 / p, s)$ - внутренняя точка “меньиего" треугольника $\Delta N_{j}$; числа $s_{j}, \widetilde{s}_{j}, s, p, q_{j}, \widetilde{q}_{j}, q$ удовлетворяют условиям теоремы 1 и для некоторого вектора $\bar{\mu} \in H_{+}^{2}$ верно равенство

$$
\left(F_{\left(N_{j}\right), \widetilde{q}_{j}}\right)_{\bar{\mu}, q}=B_{p, q,(q)}^{s} .
$$

Тогда найдется вектор $\bar{\theta} \in H_{+}^{2}$ такой, что

$$
\left(F_{\left(M_{j}\right), q_{j}}\right)_{\bar{\theta}, q}=B_{p, q,(q)}^{s} .
$$

ДокАЗАТЕЛЬСТво. По теореме С

$$
\left(\left(F_{\left(N_{0}\right), \widetilde{q}_{0}}, F_{\left(M_{2}\right), q_{2}}\right)_{\eta, q},\left(F_{\left(N_{1}\right), \widetilde{q}_{1}}, F_{\left(M_{2}\right), q_{2}}\right)_{\eta, q}\right)_{\lambda, q}=\left(F_{\left(N_{j}\right), \widetilde{q}_{j}}\right)_{\bar{\mu}, q}=B_{p, q,(q)}^{s}
$$

при $\eta=\mu_{2}, \lambda=\mu_{1} /\left(\mu_{0}+\mu_{1}\right)$. Что представляют собой пространства

$$
\left(F_{\left(N_{i}\right), \tilde{q}_{i}}, F_{\left(M_{2}\right), q_{2}}\right)_{\eta, q}, \quad i=0,1 ?
$$

Здесь возможны разные случаи:
a) $\widetilde{s}_{i} \neq s_{2}, \widetilde{p}_{i} \neq p_{2}$;
b) $\widetilde{s}_{i}=s_{2}, \widetilde{p}_{i} \neq p_{2}$;
c) $\widetilde{s}_{i} \neq s_{2}, \widetilde{p}_{i}=p_{2}$. 
В случае а) можно использовать формулу [5]

$$
\left(F_{r_{0}, q_{0}}^{t_{0}}, F_{r_{1}, q_{1}}^{t_{1}}\right)_{\alpha, q}=B L_{r, q}^{t, k}
$$

где $t=(1-\alpha) t_{0}+\alpha t_{1}, 1 / r=(1-\alpha) / t_{0}+\alpha / t_{1}, k-$ угловой коэффициент прямой, проходящей через точки $\left(1 / r_{i}, t_{i}\right)$.

Доказательство в случаях b) и с) аналогично, только вместо (1) используются формулы $(E)$ (для b) ) и $(D)$ (для с)).

Известно [5], что $B L_{p, 1}^{s, k} \subset F_{p, q}^{s} \subset B L_{p, \infty}^{s, k}$ при $1 \leqslant q \leqslant \infty$. Применяя теорему о реитерации с $\alpha=\left|M_{i} N_{i}\right| /\left|M_{i} M_{2}\right|$, из равенства

$$
\left(F_{\left(M_{i}\right), q_{i}}, F_{\left(M_{2}\right), q_{2}}\right)_{\alpha, r}=B L_{\widetilde{p}_{i}, r}^{\widetilde{s}_{i}, k}
$$

получим

$$
\left(F_{\left(M_{i}\right), q_{i}}, F_{\left(M_{2}\right), q_{2}}\right)_{\gamma, q}=\left(F_{\left(N_{i}\right), \widetilde{q}_{i}}, F_{\left(M_{2}\right), q_{2}}\right)_{\eta, q}
$$

при $\gamma=(1-\eta) \alpha+\eta$. Треугольники $\Delta M_{j}$ и $\Delta N_{j}$ гомотетичны, поэтому $\alpha$ не зависит от $i$. Из равенства (2) следует

$$
\begin{aligned}
\left(F_{\left(M_{j}\right), q_{j}}\right)_{\bar{\theta}, q} & =\left(\left(F_{\left(M_{0}\right), q_{0}}, F_{\left(M_{2}\right), q_{2}}\right)_{\gamma, q},\left(F_{\left(M_{1}\right), q_{1}}, F_{\left(M_{2}\right), q_{2}}\right)_{\gamma, q}\right)_{\lambda, q} \\
& =\left(\left(F_{\left(N_{0}\right), \widetilde{q}_{0}}, F_{\left(M_{2}\right), q_{2}}\right)_{\eta, q},\left(F_{\left(N_{1}\right), \widetilde{q}_{1}}, F_{\left(M_{2}\right), q_{2}}\right)_{\eta, q}\right)_{\lambda, q}=B_{p, q,(q)}^{s}
\end{aligned}
$$

при $\bar{\theta}=\left(\theta_{0}, \theta_{1}, \theta_{2}\right), \theta_{2}=\gamma, \theta_{1}=(1-\gamma) \lambda, \theta_{0}=(1-\gamma)(1-\lambda)$.

Допустим, применив функтор Спарра, мы получили пространство $B_{p, q,(q)}^{s}$. Будут ли выполняться нужные соотношения между параметрами $s, s_{j}, p, p_{j}, \bar{\theta}$ ? Ответ дает следующая лемма.

ЛЕмма 2. Если для данного треугольника $\Delta M_{j}\left(1 / p_{j}, s_{j}\right)$ выполнены условия теоремы 1 u

$$
\left(F_{p_{j}, q_{j}}^{s_{j}}\right)_{\bar{\theta}, q}=B_{p, q,(q)}^{s},
$$

mo

$$
s=\sum_{j=0}^{2} \theta_{j} s_{j}, \quad \frac{1}{p}=\sum_{j=0}^{2} \frac{\theta_{j}}{p_{j}} .
$$

ДокАЗАТЕЛЬСтво. Воспользуемся формулой (1). На первом этапе мы получаем пространства $B L_{\widetilde{p}, q}^{\widetilde{s}, k}$, или $F_{\tilde{p}, q}^{\widetilde{s}}$, или $B_{\widetilde{p}, q}^{\widetilde{s}}$. В любом случае выполняются равенства

$$
\widetilde{s}_{i}=(1-\eta) s_{i}+\eta s_{2}, \quad \frac{1}{\widetilde{p}_{i}}=\frac{1-\eta}{p_{0}}+\frac{\eta}{p_{1}}, \quad i=0,1 .
$$

Затем применяем функтор $(\cdot, \cdot)_{\lambda, q}$. При этом должны вьполняться условия $s=$ $(1-\lambda) \widetilde{s}_{0}+\lambda \widetilde{s}_{1}$ и $1 / p=(1-\lambda) / \widetilde{p}_{0}+\lambda / \widetilde{p}_{1}$. Проверим справедливость равенства $(3)$ для $s$ :

$$
\begin{aligned}
s & =(1-\lambda) \widetilde{s}_{0}+\lambda \widetilde{s}_{1}=(1-\lambda)(1-\eta) s_{0}+(1-\lambda) \eta s_{2}+\lambda(1-\eta) s_{1}+\eta \lambda s_{2} \\
& =(1-\lambda)(1-\eta) s_{0}+\lambda(1-\eta) s_{1}+\eta s_{2} .
\end{aligned}
$$

Выразим $\eta$ и $\lambda$ через $\theta_{j}$. Имеем $\eta=\theta_{2}, \lambda=\theta_{1} /\left(\theta_{0}+\theta_{1}\right)$, при этом $\theta_{0}+\theta_{1}+\theta_{2}=1$. Отсюда можно найти $\theta_{0}=(1-\lambda)(1-\eta), \theta_{1}=\lambda(1-\eta), \theta_{2}=\eta$. Поэтому $s=\theta_{0} s_{0}+\theta_{1} s_{1}+\theta_{2} s_{2}$. 


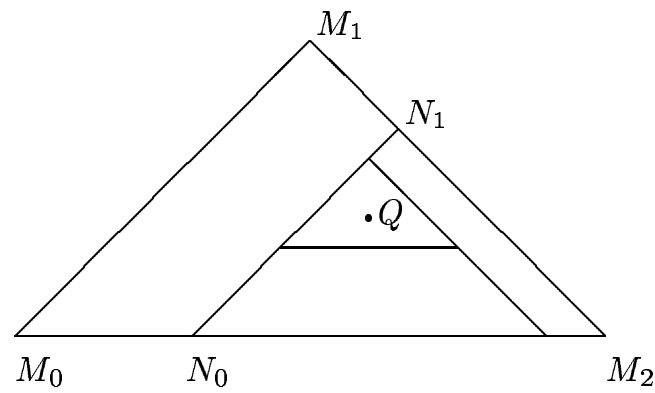

Рис. 1

ДоКАЗАТЕЛЬСТво ТЕОРЕмЫ 1 . Лемма 1 позволяет доказьвать теорему не обязательно для исходного треугольника, а для любого треугольника, гомотетичного исходному относительно одной из вершин и содержащего точку $Q$. Применяя лемму 1 трижды с гомотетией относительно каждой из вершин, мы можем показать, что достаточно доказать теорему для треугольника сколь угодно малых размеров (рис. 1).

На следующем этапе доказательства мы будем использовать теорему С, позволяющую представить функтор Спарра как композицию “одномерных" функторов Петре. $\mathrm{K}$ сожалению, классы пространств Бесова и Лизоркина-Трибеля нестабильны относительно одномерных функторов. Так при интерполяции пространств $F_{p, q}^{s}$ по наклонному отрезку $\left(p_{1} \neq p_{2}, s_{1} \neq s_{2}\right)$ мы получаем пространства $B L_{p, q}^{s, k}$, которые совпадают с пространствами Бесова в “диагональном" случае $p=q$. Другой удобный для функторов Петре частньй случай мы имеем при интерполяции по вертикальному отрезку $\left(p_{1}=p_{2}\right)$.

Поэтому будет рассмотрен вспомогательный прямоугольный треугольник $\Delta R_{i}$, при интерполяции вдоль сторон которого мы будем иметь дело с этими частными случаями.

Возможны два случая:

a) $q \neq p$;

b) $q=p$.

Рассмотрим сначала случай а). Если $q \neq p$, то можно, выбрав треугольник $\Delta M_{j}$ достаточно малым, найти прямоугольньй треугольник $\Delta R_{j}\left(1 / r_{j}, u_{j}\right), j=0,1,2$, обладающий следующими свойствами. Точки $M_{j}$ являются внутренними точками $\Delta R_{j}$, катеты треугольника $\Delta R_{j}$ параллельны осям координат, гипотенуза содержит точку $Q_{1}(1 / q, s)$ и $1<r_{j}<\infty$. Выберем числа $\eta$ и $\lambda$ таким образом, чтобы $s=(1-\eta) u_{0}+\eta u_{2}$, $1 / q=(1-\eta) / r_{1}+\eta / r_{2}$ и $1 / p=(1-\lambda) / r_{2}+\lambda / q$ (рис. 2$)$. В этом случае по теореме $\mathrm{C}$

$$
\left(F_{\left(R_{i}\right), q_{i}}\right)_{\bar{\theta}^{*}, q}=\left(\left(F_{r_{2}, q_{0}}^{u_{0}}, F_{r_{2}, q_{2}}^{u_{2}}\right)_{\eta, q},\left(F_{r_{1}, q_{1}}^{u_{1}}, F_{r_{2}, q_{2}}^{u_{2}}\right)_{\eta, q}\right)_{\lambda, q},
$$

где $\bar{\theta}^{*}=\left(\theta_{0}^{*}, \theta_{1}^{*}, \theta_{2}^{*}\right), \theta_{0}^{*}=(1-\eta)(1-\lambda), \theta_{1}^{*}=\lambda(1-\eta), \theta_{2}^{*}=\eta$.

Учитывая, что $r_{0}=r_{2}=r$ и $s=(1-\eta) u_{0}+\eta u_{2}$, с помощью формулы $(D)$ можно записать

$$
\left(F_{r, q_{0}}^{u_{0}}, F_{r, q_{2}}^{u_{2}}\right)_{\eta, q}=B_{r, q}^{s} .
$$

Так как $1 / q=(1-\eta) / r_{1}+\eta / r_{2}$ и $s=(1-\eta) u_{0}+\eta u_{2}$, из формулы $(F)$ следует

$$
\left(F_{r_{1}, q_{1}}^{u_{1}}, F_{r_{2}, q_{2}}^{u_{2}}\right)_{\eta, q}=B_{q, q}^{s}
$$




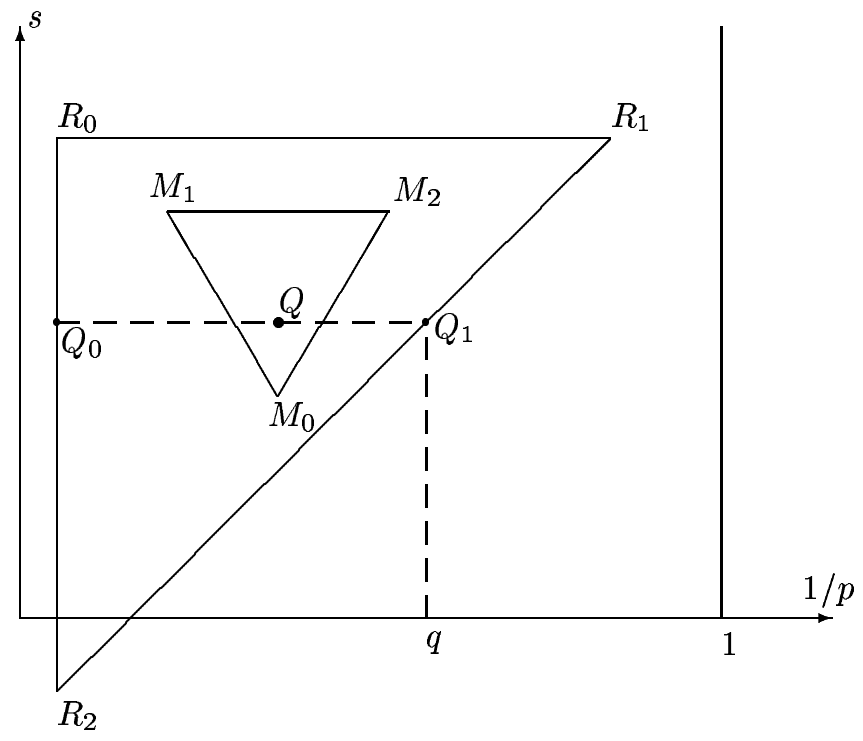

Рис. 2

На втором этапе интерполяции по формуле (4) получаем пространство

$$
\left(B_{r, q}^{s}, B_{q, q}^{s}\right)_{\lambda, q}=B_{p, q,(q)}^{s} .
$$

Итак, $\left(F_{\left(R_{i}\right), q_{i}}\right)_{\bar{\theta}^{*}, q}=B_{p, q,(q)}^{s}$. Мы получили нужное утверждение, но для вспомогательного треугольника $\Delta R_{j}$. Докажем, что $\left(F_{\left(M_{j}\right), q_{j}}\right)_{\bar{\theta}, q}=B_{p, q,(q)}^{s}$. Чтобы перейти к треугольнику $\Delta M_{j}$, надо воспользоваться теоремой А о стабильности. Для этого докажем вложения

$$
\left(F_{\left(R_{i}\right), w_{i}}\right)_{\bar{\theta}^{j}, 1} \subset F_{\left(M_{j}\right), q_{j}} \subset\left(F_{\left(R_{i}\right), w_{i}}\right)_{\bar{\theta}^{j}, \infty},
$$

где $\bar{\theta}^{j}=\left(\theta_{0}^{j}, \theta_{1}^{j}, \theta_{2}^{j}\right), M_{j}=M_{j}\left(1 / p_{j}, s_{j}\right), s_{j}=\sum_{i=0}^{2} \theta_{i}^{j} w_{i}, 1 / p_{j}=\sum_{i=0}^{2} \theta_{i}^{j} / r_{i}$. Докажем эти вложения хотя бы для $j=0$. Положим $\lambda=\theta_{1}^{0} /\left(\theta_{0}^{0}+\theta_{1}^{0}\right), \eta=\theta_{2}^{0}$ и $w=1$ или $\infty$. Тогда $\left(F_{\left(R_{0}\right), w_{0}}, F_{\left(R_{2}\right), w_{2}}\right)_{\eta, w}=\left(F_{r, w_{0}}^{u_{0}}, F_{r, w_{2}}^{u_{2}}\right)_{\eta, w}=B_{r, w}^{s_{0}}$ при $s_{0}=(1-\eta) u_{0}+\eta u_{1}$ (формула $(D))$. Хорошо известны вложения $B_{r, 1}^{s_{0}} \subset F_{r, q_{0}}^{s_{0}} \subset B_{r, \infty}^{s_{0}}$. Интерполируя по отрезку $R_{1} R_{2}$, получим равенство $\left(F_{\left(R_{1}\right), w_{1}}, F_{\left(R_{2}\right), w_{2}}\right)_{\eta, w}=B L_{r^{*}, w}^{s_{0}, k}[6]$, где $1 / r^{*}=$ $(1-\eta) / r_{1}+\eta / r_{2}$. Известно, что при $1 \leqslant q_{0} \leqslant \infty$ справедливы вложения $B L_{r^{*}, 1}^{s_{0}, k} \subset$ $F_{r^{*}, q_{0}}^{s_{0}} \subset B L_{r^{*}, \infty}^{s_{0}, k}$. Поэтому

$$
\begin{aligned}
\left(F_{\left(R_{i}\right), w_{i}}\right)_{\bar{\theta}^{0}, 1} & =\left(B_{r, 1}^{s_{0}}, B L_{r^{*}, 1}^{s_{0}, k}\right)_{\lambda, 1} \subset\left(F_{r, q_{0}}^{s_{0}}, F_{r^{*}, q_{0}}^{s_{0}}\right)_{\lambda, q_{0}} \\
& \subset\left(B_{r, \infty}^{s_{0}}, B L_{r^{*}, \infty}^{s_{0}, k}\right)_{\lambda, \infty}=\left(F_{\left(R_{i}\right), w_{i}}\right)_{\bar{\theta}^{0}, \infty} .
\end{aligned}
$$

Так как $\left(F_{r, q_{0}}^{s_{0}}, F_{r^{*}, q_{0}}^{s_{0}}\right)_{\lambda, q_{0}}=F_{p_{0}, q_{0}}^{s_{0}}$, то отсюда следует вложение (5). По теореме А

$$
\left(\left(F_{\left(R_{0}\right), w_{0}}\right)_{\bar{\theta}^{0}, w},\left(F_{\left(R_{1}\right), w_{1}}\right)_{\bar{\theta}^{1}, w},\left(F_{\left(R_{2}\right), w_{2}}\right)_{\bar{\theta}^{2}, w}\right)_{\bar{\theta}, q}=\left(F_{\left(R_{i}\right), w_{i}}\right)_{\bar{\theta}^{*}, q}=B_{p, q,(q)}^{s}
$$

независимо от $w$. Поэтому из вложений (5) следует равенство

$$
\left(F_{\left(M_{j}\right), q_{j}}\right)_{\bar{\theta}, q}=B_{p, q,(q)}^{s} .
$$


Таким образом, теорема доказана при условии, что $p \neq q$.

Разберем случай b), когда $p=q$. Для доказательства выберем две точки $Q_{1}\left(1 / r_{1}, s\right)$, $Q_{2}\left(1 / r_{2}, s\right)$, принадлежащие внутренности треугольника $\Delta M_{j}$, так, чтобы $r_{1}<p<r_{2}$. Так как $q \neq r_{1}$ и $q \neq r_{2}$, то при соответствующих $\bar{\theta}^{i} \in H_{+}^{2}$ справедливо равенство $\left(F_{\left(M_{j}\right), q_{j}}\right)_{\bar{\theta}^{i}, q}=B_{\left(Q_{i}\right), q,(q)}$. Тогда можно выбрать $\lambda \in(0,1)$ такое, что

$$
\left(B_{\left(Q_{1}\right), q,(q)}, B_{\left(Q_{2}\right), q,(q)}\right)_{\lambda, q}=B_{p, q,(q)}^{s} .
$$

С помощью теоремы В получаем равенство

$$
\left(F_{\left(M_{j}\right), q_{j}}\right)_{\bar{\theta}, q}=\left(\left(F_{\left(M_{j}\right), q_{j}}\right)_{\bar{\theta}^{1}, q},\left(F_{\left(M_{j}\right), q_{j}}\right)_{\bar{\theta}^{2}, q}\right)_{\lambda, q}=B_{p, q,(q)}^{s} .
$$

СледСтвиЕ. Пусть $1<p_{i}<\infty, 1<q_{i}<\infty, 0<\lambda<1 u 1 / q=(1-\lambda) / q_{0}+\lambda / q_{1}$, $1 / p=(1-\lambda) / p_{0}+\lambda / p_{1}, s=(1-\lambda) s_{0}+\lambda s_{1}$. Тогдa

$$
\left(B_{p_{0}, q_{0},\left(q_{0}\right)}^{s_{0}}, B_{p_{1}, q_{1},\left(q_{1}\right)}^{s_{1}}\right)_{\lambda, q}=B_{p, q,(q)}^{s} .
$$

Разберем отдельно случай, когда $q=1$ или $\infty$.

Теорема 2. Если величины $s_{j}, p_{j}, q_{j}, j=0,1,2$, удовлетворяют условиям теоремы 1, то справедливы вложения

$$
B_{p, 1,(1)}^{s} \subset\left(F_{p_{j}, q_{j}}^{s_{j}}\right)_{\bar{\theta}, 1} \subset F_{p, 1,(1)}^{s}, \quad F_{\infty,(\infty)}^{s} \subset\left(F_{p_{j}, q_{j}}^{s_{j}}\right)_{\bar{\theta}, \infty} \subset B_{p, \infty,(\infty)}^{s} .
$$

ДокАЗАТЕЛЬСТво. Рассмотрим вспомогательный треугольник $\Delta R_{i}\left(1 / r_{i}, u_{i}\right), i=$ $0,1,2$, для которого точки $\left(1 / p_{j}, s_{j}\right)$ являются внутренними, $1<r_{i}<\infty$ и $u_{0}=u_{1}$. Тогда можно указать числа $\eta \in(0,1)$ и $\lambda \in(0,1)$ такие, что

$$
\left(F_{p_{j}, q_{j}}^{s_{j}}\right)_{\bar{\theta}, q}=\left(\left(F_{r_{0}, q_{0}^{*}}^{u_{0}}, F_{r_{2}, q_{2}^{*}}^{u_{2}}\right)_{\eta, q},\left(F_{r_{1}, q_{1}^{*}}^{u_{1}}, F_{r_{2}, q_{2}^{*}}^{u_{2}}\right)_{\eta, q}\right)_{\lambda, q} .
$$

Заметим, что $\left(F_{r_{i}, q_{i}^{*}}^{u_{i}}, F_{r_{2}, q_{2}^{*}}^{u_{2}}\right)_{\eta, 1}=B L_{r, 1}^{s, k}[6]$. Вообще говоря, известно, что $B L_{p, 1}^{s, k} \neq$ $B_{p, 1}^{s}$ и $B L_{p, 1}^{s, k} \not \subset B_{p, 1}^{s}[6]$, но $B_{p, 1,(1)}^{s} \subset B L_{p, 1}^{s, k} \subset F_{p, 1}^{s}[6]$, [7]. Так как $\left(B_{r_{0}, 1,(1)}^{s}, B_{r_{1}, 1,(1)}^{s}\right)_{\lambda, 1}$ $=B_{p, 1,(1)}^{s}$ и $\left(F_{r_{0}, 1}^{s}, F_{r_{1}, 1}^{s}\right)_{\lambda, 1}=F_{p, 1,(1)}^{s}$, то

$$
B_{p, 1,(1)}^{s} \subset\left(F_{r_{i}, q_{i}}^{u_{i}}\right)_{\bar{\theta}^{*}, 1} \subset F_{p, 1,(1)}^{s} .
$$

Используя теорему А, можно заключить, что $\left(F_{r_{i}, q_{i}^{*}}^{u_{i}}\right)_{\bar{\theta}^{*}, 1}=\left(F_{p_{j}, q_{j}}^{s_{j}}\right)_{\bar{\theta}, 1}$. Следовательно,

$$
B_{p, 1,(1)}^{s} \subset\left(F_{p_{j}, q_{j}}^{s_{j}}\right)_{\bar{\theta}, 1} \subset F_{p, 1,(1)}^{s} .
$$

Аналогично,

$$
F_{p, \infty,(\infty)}^{s} \subset\left(F_{p_{j}, q_{j}}^{s_{j}}\right)_{\bar{\theta}, \infty} \subset B_{p, \infty,(\infty)}^{s} .
$$

3. Пространства Бесова. Для пространств Бесова типа $B_{p, q,(q)}^{s}$ докажем интерполящионную теорему. 
ТЕОрема 3. Пусть точки $M_{j}\left(1 / p_{j}, s_{j}\right), j=0,1, \ldots, m, m>2$, образуют выпуклый многоугольник. Предположим, что никакие три из них не лежат на одной прямой и $1<p_{j}<\infty, 1<q_{j}<\infty, \bar{\lambda} \in H_{+}^{m}, s=\sum_{j=0}^{m} \lambda_{j} s_{j}, 1 / p=\sum_{j=0}^{m} \lambda_{j} / p_{j}$. тогда

$$
\left(B_{p_{j}, q_{j},\left(q_{j}\right)}^{s_{j}}\right)_{\bar{\lambda}, q}=B_{p, q,(q)}^{s} .
$$

ДокАЗАТЕЛЬСТво. Для данного многоугольника найдется треугольник $\Delta R_{i}\left(1 / r_{i}\right.$, $u_{i}$ ) такой, что $M_{j}$ - внутренние точки этого треугольника и $1<r_{i}<\infty$. Тогда по теореме 1 можно указать векторы $\bar{\theta}^{j} \in H_{+}^{2}$ такие, что при любых $v_{i} \in[1, \infty] B_{p_{j}, q_{j},\left(q_{j}\right)}^{s_{j}}=$ $\left(F_{\left(R_{i}\right), v_{i}}\right)_{\bar{\theta}^{j}, q_{j}}$. По теореме $\mathrm{A}$

$$
\left(B_{p_{j}, q_{j},\left(q_{j}\right)}^{s_{j}}\right)_{\bar{\lambda}, q}=\left(\left(F_{\left(R_{i}\right), v_{i}}\right)_{\bar{\theta}^{j}, q_{j}}\right)_{\bar{\lambda}, q}=\left(F_{\left(R_{i}\right), v_{i}}\right)_{\bar{\theta}, q}=B_{p, q,(q)}^{s},
$$

если $\bar{\theta}=\sum_{j=0}^{m} \lambda_{j} \bar{\theta}^{j}$.

Сформулируем интерполяционную теорему для пространств Бесова, использующую слабые условия вида $T: F_{(M), 1,(1)} \rightarrow F_{(N), \infty,(\infty)}$.

ТЕОРема 4. Пусть заданы два невырохсенных треугольника $\Delta M_{j}\left(1 / p_{j}, s_{j}\right)$, $\Delta N_{j}\left(1 / r_{j}, u_{j}\right)$ и вектор $\bar{\theta} \in H_{+}^{2}$. Для линейного оператора $T$ выполняются слабые условия $T: F_{\left(M_{j}\right), 1,(1)} \rightarrow F_{\left(N_{j}\right), \infty,(\infty)}, j=0,1,2,1 / p=\sum_{j=0}^{2} \theta_{j} / p_{j}, s=\sum_{j=0}^{2} \theta_{j} s_{j}$, $1 / r=\sum_{j=0}^{2} \theta_{j} / r_{j}, u=\sum_{j=0}^{2} \theta_{j} u_{j}$. Тогда оператор $T: B_{p, q,(q)}^{s} \rightarrow B_{r, q,(q)}^{u}$. Ecлu, кроме того, выполняется условие $r \geqslant p$, то оператор $T: \stackrel{B}{p}_{p}^{s} \rightarrow B_{r}^{u}$.

4. Связь между функторами Спарра и Петре. Все приведенные выше доказательства можно было бы существенно упростить, если вместо теоремы С мы имели бы более простую формулу для связи между функторами Спарра и Петре. В качестве гипотезы можно рассмотреть формулу

$$
(\bar{A})_{\bar{\theta}, q}=\left(\left(A_{0}, A_{1}\right)_{\eta, q},\left(A_{2}, A_{3}\right)_{\eta, q}\right)_{\lambda, q}
$$

при $n=3, \eta=\theta_{1}+\theta_{3}, \lambda=\theta_{2}+\theta_{3}$. Из результатов данной статьи и [7] вытекает, что это равенство действительно имеет место при $A_{i}=F_{p_{i}, q_{i}}^{s_{i}}$ или $B_{p_{i}}^{s_{i}}, q_{i}$, где $s_{0}=s_{1}, s_{2}=s_{3}$, $p_{0}=p_{2}, p_{1}=p_{3}, 1<q<\infty$. Однако равенство (6) справедливо не всегда. В статье [2] приведен соответствуюший контрпример. Показано, что для метода Фернандеса ( "многомерный" интерполяционньй метод, аналогичньй методу Спарра [8]) отсюда следует отношение $(\bar{A})_{\bar{\theta}, q ; K, \Phi е р н} \neq(\bar{A})_{\bar{\theta}, q ; J, \Phi е р н}$. Здесь рассматривается пример, в котором справедливо равенство $(\bar{A})_{\bar{\theta}, q ; K, \text { Спарр }}=(\bar{A})_{\bar{\theta}, q ; J, \text { Спарр }}$, но формула $(6)$ неверна.

ПРИмеР. Пусть $A_{0}=L_{p_{0}}\left[\ell_{r}^{s_{0}}\right]\left(\mathbb{R}^{1}\right), A_{1}=L_{p_{1}}\left[\ell_{r}^{s_{1}}\right]\left(\mathbb{R}^{1}\right), A_{2}=A_{3}=L_{p_{2}}\left[\ell_{r}^{s_{2}}\right]\left(\mathbb{R}^{1}\right)$, $\bar{\theta} \in H_{+}^{3}, 1<p_{0}, p, p_{1}<\infty, q=p, p_{2} \neq p, \eta=\theta_{1}+\theta_{2}, \lambda=\theta_{2}+\theta_{3}, 1 / p=(1-\eta) / p_{0}+\eta / p_{1}$, $s=(1-\lambda) s_{0}+\lambda s_{1}$.

Легко видеть, что функтор Спарра не зависит от порядка нумерации пространств $A_{1}, A_{2}, A_{3}$, т.е.

$$
\left(A_{0}, A_{1}, A_{2}, A_{3}\right)_{\bar{\theta}, q}=\left(A_{0}, A_{2}, A_{1}, A_{3}\right)_{\bar{\theta}^{*}, q}
$$


где $\bar{\theta}^{*}=\left(\theta_{0}, \theta_{2}, \theta_{1}, \theta_{3}\right)$. Применим формулу $(6)$ к правой части этого равенства. Тогда

$$
\left(A_{0}, A_{2}, A_{1}, A_{3}\right)_{\bar{\theta}^{*}, q}=\left(\left(A_{0}, A_{2}\right)_{\eta^{*}, q},\left(A_{1}, A_{3}\right)_{\eta^{*}, q}\right)_{\lambda^{*}, q^{\prime}},
$$

где $\eta^{*}=\theta_{2}+\theta_{3}=\lambda, \lambda^{*}=\theta_{1}+\theta_{3}=\eta$, т.е. если формула (6) верна, то должно выполняться равенство

$$
\left(\left(A_{0}, A_{1}\right)_{\eta, q},\left(A_{2}, A_{3}\right)_{\eta, q}\right)_{\lambda, q}=\left(\left(A_{0}, A_{2}\right)_{\lambda, q},\left(A_{1}, A_{3}\right)_{\lambda, q}\right)_{\eta, q} .
$$

Покажем, что в нашем случае это неверно. Так как $A_{2}=A_{3}$, то по формуле $(C)$

$$
\left(\left(A_{0}, A_{2}\right)_{\lambda, q},\left(A_{1}, A_{3}\right)_{\lambda, q}\right)_{\eta, q}=\left(\left(A_{0}, A_{2}\right)_{\lambda, q},\left(A_{1}, A_{2}\right)_{\lambda, q}\right)_{\eta, q}=\left(A_{0}, A_{1}, A_{2}\right)_{\bar{\nu}, q},
$$

где $\nu_{0}=(1-\lambda)(1-\eta), \nu_{1}=\eta(1-\lambda), \nu_{2}=\lambda$. Заметим, что все формулы для интерполяции пар пространств $\left(F_{p_{0}}^{s_{0}}, q_{0}, F_{p_{1}, q_{1}}^{s_{1}}\right)$ имеют аналоги для пространств $L_{p_{i}}\left[\ell_{q_{i}}^{s_{i}}\right]$. При этом вместо утверждения теоремы 1 мы получаем равенство $\left(L_{p_{i}}\left[\ell_{q_{i}}^{s_{i}}\right]\right)_{\bar{\theta}, q}=\ell_{q}^{s}\left[L_{p, q}\right]$. В нашем случае это означает, что $\left(A_{0}, A_{1}, A_{2}\right)_{\bar{\nu}, q}=\ell_{q}^{s}\left[L_{p^{*}, q}\right]$, где $1 / p^{*}=\sum_{i=0}^{2} \nu_{i} / p_{i}$.

Найдем пространства $\left(\left(A_{0}, A_{1}\right)_{\eta, q},\left(A_{2}, A_{3}\right)_{\eta, q}\right)_{\lambda, q}$. На первом этапе

$$
\left(A_{0}, A_{1}\right)_{\eta, q}=\left(L_{p_{0}}\left[\ell_{r}^{s_{0}}\right], L_{p_{1}}\left[\ell_{r}^{s_{0}}\right]\right)_{\lambda, q}=L_{q}\left[\ell_{r}^{s_{0}}\right]
$$

так как $q=p=(1-\lambda) / p_{0}+\lambda / p_{1}$, и

$$
\left(A_{2}, A_{3}\right)_{\eta, q}=A_{2}=L_{p_{2}}\left[\ell_{r}^{s_{1}}\right]
$$

так как $A_{2}=A_{3}$.

Осталось найти $\left(L_{q}\left[\ell_{r}^{s_{0}}\right], L_{p_{2}}\left[\ell_{r}^{s_{1}}\right]\right)_{\lambda, q}$. Получаются пространства [6]

$$
L_{p^{*}, q}^{s, k}:=L_{p^{*}, q}\left(2^{j\left(s-k / p^{*}\right)}, 2^{j k} \nu \times m_{1}\right),
$$

т.е. пространства Лоренца на $\mathbb{Z}_{+} \times \mathbb{R}_{1}$ с весом $2^{j\left(s-k / p^{*}\right)}$ и мерой $2^{j k} \nu \times m_{1}, \nu$-атомическая мера на $\mathbb{Z}_{+}=\{0,1,2, \ldots\}$ с мерой атома, равной единице, $k$ - угловой коэффициент прямой, проходящей через точки $\left(1 / p, s_{0}\right)$ и $\left(1 / p_{2}, s_{1}\right)$,

$$
\frac{1}{p^{*}}=\frac{1-\lambda}{q}+\frac{\lambda}{p_{2}} \neq \frac{1}{p}, \quad s=(1-\lambda) s_{0}+\lambda s_{1} .
$$

Докажем, что $\ell_{q}^{s}\left[L_{p^{*}, q}\right] \neq L_{p^{*}, q}^{s, k}$. Для этого рассмотрим последовательность функций $h_{n}=\left(h_{j}^{(n)}\right)_{j=0}^{\infty}$,

$$
h_{j}^{(n)}= \begin{cases}2^{-j\left(s-k / p^{*}\right)} \chi_{\left[0,2^{-j k}\right]}(x) & \text { при } j \leqslant n, \\ 0 & \text { при } j>n,\end{cases}
$$

определенных на $\mathbb{Z}_{+} \times \mathbb{R}_{1}, n, j \in \mathbb{Z}_{+}, x \in \mathbb{R}_{1}$. Оценим нормы

$$
\begin{aligned}
\left\|h_{n} \mid L_{p^{*}, q}^{s, k}\right\| & =\left(\int_{0}^{\infty}\left(t^{1 / p^{*}}\left(\left\{\chi_{\left[0,2^{-j k}\right]}(x) 2^{j\left(s-k / p^{*}\right)} 2^{-j\left(s-k / p^{*}\right)}\right\}_{j=0}^{n}\right)^{*}\right)^{q} \frac{d t}{t}\right)^{1 / q} \\
& =\left(\int_{0}^{\infty}\left(t^{1 / p^{*}} \chi_{[0, n]}(t)\right)^{q} \frac{d t}{t}\right)^{1 / q}=\left(\int_{0}^{n} t^{q / p^{*}-1} d t\right)^{1 / q} \\
& =C\left(t^{q / p^{*}} \mid{ }_{0}^{n}\right)^{1 / q}=C n^{1 / p^{*}}, \\
\left\|h_{n} \mid \ell_{q}^{s}\left[L_{p, q}\right]\right\| & =C\left\|\left\{2^{-j\left(s-k / p^{*}\right)} 2^{-j k / p^{*}}\right\}_{j=0}^{n}\left|\ell_{q}^{s}\|=C\|\left\{2^{-j s} 2^{j s}\right\}_{j=0}^{n}\right| \ell_{q}\right\|=C n^{1 / q} .
\end{aligned}
$$


Tак как $q=p \neq p^{*}$, то

$$
\lim _{n \rightarrow \infty} \frac{\left\|h_{n} \mid L_{p^{*}, q}^{s, k}\right\|}{\left\|h_{n} \mid \ell_{q}^{s}\left[L_{p^{*}, q}\right]\right\|}=0 \text { или } \infty .
$$

Следовательно,

$$
\left(\left(A_{0}, A_{1}\right)_{\eta, q},\left(A_{2}, A_{3}\right)_{\eta, q}\right)_{\lambda, q} \neq\left(\left(A_{0}, A_{2}\right)_{\lambda, q},\left(A_{1}, A_{3}\right)_{\lambda, q}\right)_{\eta, q}
$$

и формула (6) в данном случае неверна.

\section{СПИСОК ЦИТИРОВАННОЙ ЛИТЕРАТУРЫ}

[1] Sparr G. Interpolation of several Banach spaces // Ann. Mat. Pura Appl. 1976. V. 99. P. 247-316.

[2] Cobos F., Peetre J. Interpolation of compact operators: the multidimensional case // Proc. London Math. Soc. Ser. 3. 1991. V. 63. P. 371-400.

[3] Carro M. J. Real interpolation for families of Banach spaces // Studia Math. 1994. V. 59. №1. P. 1-21.

[4] Asekritova I., Krugljak N. On equivalence of $K$ - and $J$-methods for $(n+1)$-tuples of Banach spaces // Studia Math. 1997. V. 122. № 2. P. 99-115.

[5] Трибель Х. Теория интерполяции, функциональные пространства, дифференциальные операторы. М.: Мир, 1980.

[6] Крепкогорский В.Л. Интерполяция в пространствах Лизоркина-Трибеля и Бесова // Матем. сб. 1994. Т. 185. № 7. С. 63-76.

[7] Крепкогорский В. Л. О многомерных методах интерполяции // Изв. вузов. Матем. 1999. №11. C. 41-49.

[8] Fernandez D. L. Interpolation of $2^{d}$ Banach spaces and the Calderon space $X(E) / /$ Proc. London Math. Soc. Ser. 3. 1988. V. 56. P. 143-162.

Казанский филиал военного артиллерийского университета 